\title{
Longitudinal transcriptome-wide gene expression analysis of sleep deprivation treatment shows involvement of circadian genes and immune pathways
}

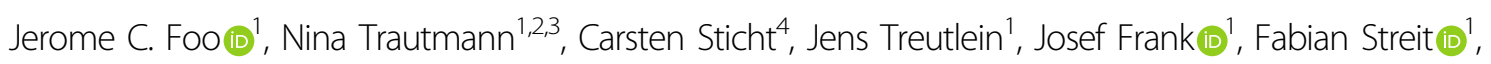
Stephanie H. Witt (1) , Carolina De La Torre ${ }^{4}$, Steffen Conrad von Heydendorff', Lea Sirignano', Junfang Chen², Bertram Müller-Myhsok ${ }^{5,6,7}$, Andreas Meyer-Lindenberg ${ }^{2}$, Christian C. Wittt ${ }^{8}$, Maria Gilles ${ }^{2}$, Michael Deuschle ${ }^{2}$ and Marcella Rietschel (10)

\begin{abstract}
Therapeutic sleep deprivation (SD) rapidly induces robust, transient antidepressant effects in a large proportion of major mood disorder patients suffering from a depressive episode, but underlying biological factors remain poorly understood. Research suggests that these patients may have altered circadian molecular genetic 'clocks' and that SD functions through 'resetting' dysregulated genes; additional factors may be involved, warranting further investigation. Leveraging advances in microarray technology enabling the transcriptome-wide assessment of gene expression, this study aimed to examine gene expression changes accompanying SD and recovery sleep in patients suffering from an episode of depression. Patients $(N=78)$ and controls $(N=15)$ underwent $S D$, with blood taken at the same time of day before SD, after one night of SD and after recovery sleep. A transcriptome-wide gene-by-gene approach was used, with a targeted look also taken at circadian genes. Furthermore, gene set enrichment, and longitudinal gene set analyses including the time point after recovery sleep, were conducted. Circadian genes were significantly affected by $\mathrm{SD}$, with patterns suggesting that molecular clocks of responders and non-responders, as well as patients and controls respond differently to chronobiologic stimuli. Notably, gene set analyses revealed a strong widespread effect of SD on pathways involved in immune function and inflammatory response, such as those involved in cytokine and especially in interleukin signalling. Longitudinal gene set analyses showed that in responders these pathways were upregulated after SD; in non-responders, little response was observed. Our findings emphasize the close relationship between circadian, immune and sleep systems and their link to etiology of depression at the transcriptomic level.
\end{abstract}

Correspondence: Jerome C. Foo (jerome.foo@zi-mannheim.de)

'Department of Genetic Epidemiology in Psychiatry, Central Institute of Mental Health, Medical Faculty Mannheim, University of Heidelberg, Mannheim, Germany

2Department of Psychiatry and Psychotherapy, Central Institute of Mental Health, Medical Faculty Mannheim, University of Heidelberg, Mannheim, Germany

Full list of author information is available at the end of the article.

\section{Introduction}

Therapeutic sleep deprivation (SD) rapidly induces robust antidepressant effects in a large proportion of major mood disorder patients suffering from a depressive episode $^{1-5}$. The effects of the treatment are transient as relapse is usually observed after recovery sleep. Although $\mathrm{SD}$ is considered useful and recommended, it is rarely applied in the clinical routine ${ }^{6}$. The mechanisms through which SD exerts its antidepressant effects nevertheless

\section{(-) The Author(s) 2019}

(c) (i) Open Access This article is licensed under a Creative Commons Attribution 4.0 International License, which permits use, sharing, adaptation, distribution and reproduction cc) in any medium or format, as long as you give appropriate credit to the original author(s) and the source, provide a link to the Creative Commons license, and indicate if changes were made. The images or other third party material in this article are included in the article's Creative Commons license, unless indicated otherwise in a credit line to the material. If material is not included in the article's Creative Commons license and your intended use is not permitted by statutory regulation or exceeds the permitted use, you will need to obtain permission directly from the copyright holder. To view a copy of this license, visit http://creativecommons.org/licenses/by/4.0/. 
offer important insights into the biological factors involved in depression and antidepressant response, and have been the focus of recent research ${ }^{7-11}$. Work in humans ${ }^{12-15}$ as well as animals ${ }^{16-18}$ consistently documents the effects of mistimed or insufficient sleep and sleep deprivation on circadian gene expression (such as CLOCK, ARNTL [BMAL1], PER1, PER2, PER3, etc.) as well as on genes involved in related biological processes such as inflammatory, immune and stress response ${ }^{19,20}$. A prominent hypothesis about the antidepressant mechanism underlying $\mathrm{SD}$ is that it restores circadian rhythmicity, which is often dysregulated in depression, via resetting clock gene transcription ${ }^{21,22}$. The well-controlled nature and rapidity of response to $\mathrm{SD}$ treatment ${ }^{23}$ renders it a promising context to investigate associated biological measures such as gene expression.

While no systematic investigation of gene expression changes in depressed patients undergoing SD treatment has been conducted to date, the study of gene expression in major depressive disorder (MDD) has raised the idea that genes associated with MDD are enriched for inflammation and immune response pathways, which may be linked to the sleep disturbances observed in depres$\operatorname{sion}^{24-26}$. Circadian rhythms are found in the majority of physiological processes and the immune system is no different, with alterations of these rhythms leading to disturbed immune responses ${ }^{27}$. The immune system and circadian clock circuitry crosstalk, with immune challenges and mediators, such as cytokines, also feeding back to affect circadian rhythms ${ }^{28}$. Cytokines, including chemokines, interferons and interleukins, are integral to sleep homeostat regulation and can modulate behavioural and physiological functions ${ }^{29}$.

We recently conducted a naturalistic study, which aimed to examine clinical and genetic factors predicting response to $\mathrm{SD}^{30}$. This was conducted in a sample of major mood disorder inpatients experiencing a depressive episode $(n=78)$ and healthy controls $(n=15)$. Briefly, $72 \%$ of patients responded to SD. Responders and nonresponders did not differ in self/expert assessed symptom ratings or chronotype, but mood differed. Response was associated with lower age and later age at lifetime disease onset. Higher genetic burden of depression was observed in non-responders than healthy controls, with responders having intermediate risk scores.

The present study now aimed to examine gene expression changes accompanying SD and recovery sleep during a depressive episode using a longitudinal design, looking at changes in peripheral blood gene expression in the same sample. Gene expression changes occurring after $\mathrm{SD}$ and recovery sleep, as well as associated with response and case-control status, were explored. In a hypothesisdriven approach, expression patterns of circadian genes were investigated. For a systematic search, transcriptome- wide gene-by-gene and gene set enrichment analyses were performed, while longitudinal gene set analysis ${ }^{31}$ explored dynamics in expression trajectories at the functional gene set level.

\section{Methods and materials \\ Participants}

The present sample has been described elsewhere ${ }^{30}$. Seventy-eight inpatients (34 females; age mean \pm standard deviation $=43.54 \pm 14.80$ years) presenting with a depressive episode (unipolar, $n=71$; bipolar I, $n=6$; and bipolar II, $n=1$ ) and on stable medication for $\geq 5$ days participated in this study. Depression was diagnosed according to ICD-10 criteria. Patients were recruited from consecutive admissions to the depression unit of the Department of Psychiatry and Psychotherapy of the Central Institute of Mental Health (CIMH), Mannheim, Germany. Prescribed medication included typical and atypical antidepressants, lithium, and adjunct therapies (anticonvulsants, antipsychotics and sleeping agents). Fifteen healthy controls (eight females; $40.53 \pm 15.90$ years) with no history of psychiatric/somatic disorders were recruited through an online advertisement on the $\mathrm{CIMH}$ website. The criteria for inclusion and exclusion were the same as the criteria for patients, except controls needed to lack psychiatric diseases, which was evaluated via Structured Clinical Interview for DSM-IV Axis II disorders (SCID-II) prior to SD. The investigation was carried out in accordance with the Declaration of Helsinki and approved by the local ethics committee. All participants provided written informed consent following a detailed explanation of the study.

\section{Sleep deprivation}

On Day 1, participants gave informed consent and entered the study (see Fig. 1). SD was conducted from Day

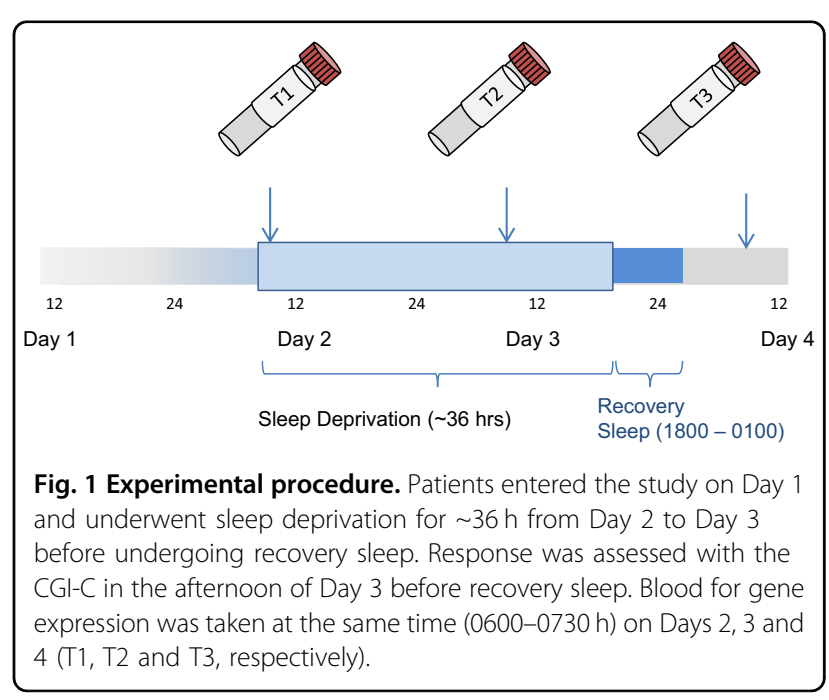


2-Day 3, in small groups of 1-5 participants under staff supervision. Participants were free to move around during the night of the SD protocol and were supervised by staff and occupied with activities such as games and walks to ensure wakefulness. Resting in bed was not allowed and SD was carried out on the ward under regular ward lighting conditions (no bright or dim lighting). Food intake was not restricted and some patients consumed snacks (i.e. bread) during SD. Participants underwent the same protocol irrespective of habitual sleep/wake timing; patients followed ward routines (i.e. lights on, lights out times) prior to inclusion to the study. Participants remained awake from $\sim 0600 \mathrm{~h}$ on Day 2 to $1800 \mathrm{~h}$ on Day 3 (36 h). On Day 3, participants underwent recovery sleep from $1800-0100 \mathrm{~h}$. Sleep phase advance was then carried out, shifting sleep $1 \mathrm{~h}$ forward each day until the patient's regular sleep pattern was reached. Controls participated alongside patients. Response was assessed by the senior clinical researcher using the Clinical Global Impression Scale for Global Improvement/Change in the afternoon on Day 3.

\section{Data collection}

Blood samples were collected in RNA-stabilizing PAXgene tubes (Qiagen, Hilden, Germany), processed according to standard procedures, and stored at $-80^{\circ} \mathrm{C}$ until analysis. Blood was collected at the same time (between 0600-0730 h) on Day 2 before SD (T1), on Day 3 after SD (T2) and on Day 4 (T3) (see Fig. 1). The number of samples decreased over time points (Table 1) due to non-participation.

\section{Sample preparation and analysis RNA and microarray analyses}

Laboratory analyses were performed using standard methods (see Supplementary Information, SI).

\section{Gene expression data pre-processing}

A Custom CDF Version 20 with ENTREZ-based gene definitions was used to annotate the Affymetrix GeneChip $^{\text {TM }}$ Human Gene 2.0 ST arrays used for gene expression profiling ${ }^{32}$. The Raw fluorescence intensity values were normalized applying quantile normalization and RMA background correction using SAS JMP11
Genomics, version 7 (SAS Institute, Cary, NC, USA). The final dataset comprised mRNA expression targeting 24,733 unique genes for each time point per participant.

\section{Data analysis}

Analyses were conducted in R (Microsoft R Open 3.4.2). Significance was set at FDR $\mathrm{q}<0.05$.

\section{Gene-based analysis}

Analyses were conducted using lme4 (Version 1.1-17). Linear mixed effects models with random intercepts were fitted to examine gene expression differences between $\mathrm{T} 1$ and T2 ('effect of time point'). Three main models were fitted: effect of time point in 'all patients' (M1), in 'responders vs. non-responders' (M2) and in 'patients vs. controls' (M3). In all analyses, age and sex were included as covariates.

Models were specified as follows: for each transcript, likelihood ratio tests were calculated between two models (h1 vs h0). In both models, gene expression was specified as the dependent variable, covariates were specified as fixed effects and the individual was specified as a random effect. h1 contained the comparison of interest specified as a fixed effect while h0 was a reduced model without it. That is, in M1, the 'effect of time point' was the only difference between h1 and h0, while in M2 and M3, the effect of interest was the interaction of the comparison group status (i.e. responder/non-responder, and case/ control) and 'effect of time point'.

Additionally, whether differences in expression levels at T1 'baseline' were informative about response and disease status was examined using linear models, controlling for sex and age.

\section{Targeted examination of differential expression of circadian genes}

To determine differential expression of circadian genes, a list of genes comprising both traditional clock genes (in order of highest signal-to-noise ratio predicting circadian rhythmicity): PER1, NR1D2, PER3, NR1D1, PER2, ARNTL, NPAS2, CLOCK, CRY2 and CRY1 as well as other top genes shown to predict circadian rhythmicity in human blood (DDIT4, CLEC4E, FKBP5, DAAM2, TPST1, IL13RA1, SMAP2, HNRNPDL, FOSL2, PER1, FLT3,

Table 1 Samples included for analysis at different time points.

\begin{tabular}{|c|c|c|c|c|c|}
\hline Time point & All subjects & Patients & Responders & Non-responders & Controls \\
\hline T1 & 91 & 76 (43M/33F) & $60(36 \mathrm{M} / 24 \mathrm{~F})$ & 16 (7M/9F) & 15 (7M/8F) \\
\hline $\mathrm{T} 2$ & 87 & $72(41 \mathrm{M} / 31 \mathrm{~F})$ & $56(34 \mathrm{M} / 22 \mathrm{~F})$ & 16 (7M/9F) & 15 (7M/8F) \\
\hline $\mathrm{T} 3$ & 81 & 66 (38M/28F) & $53(32 \mathrm{M} / 21 \mathrm{~F})$ & $13(6 \mathrm{M} / 7 \mathrm{~F})$ & 15 (7M/8F) \\
\hline Total & 259 & $214(122 \mathrm{M} / 92 \mathrm{~F})$ & 169 (102M/67F) & $45(20 M / 25 F)$ & $45(21 \mathrm{M} / 24 \mathrm{~F})$ \\
\hline
\end{tabular}


CDC42EP2, TMEM88, NR1D2, RBM3) in ref. ${ }^{33}$ was used to take a focused look at results of the gene-based analysis. Using a Monte-Carlo approach, the probability of obtaining at least the observed number of significant associations ( $p<0.05$ uncorrected) in a random gene set of the same size was calculated (see SI, Fig. S1a-c).

\section{Gene set enrichment analysis}

Gene Set Enrichment Analysis (GSEA) ${ }^{34}$ was used to determine whether differentially expressed genes offered biologically meaningful insights about SD. Ranked lists were created based on results of models M1-M3, using a signed $\log 10$ transformed $\mathrm{p}$-value with sign denoting direction of change, as described elsewhere ${ }^{35}$. To allow a concise interpretation of the potentially widespread effects of SD interventions, the Hallmark gene set collection ${ }^{36}$ (MSigDB Version 6.2), comprising 50 gene sets representing specific well-defined biological states/processes displaying coherent expression, was used. The heme metabolism gene set was excluded due to the globin interference artefact (communication with ThermoFisher).

\section{Longitudinal Gene Set Trajectory}

Time-course Gene Set Analysis (TcGSA) ${ }^{31}$ (Version 0.12.1) was employed to examine gene expression dynamics over all three time points. TcGSA, employing mixed models, detects gene sets in which expression changes over time, taking between-gene and individual variability into account, with higher sensitivity and better interpretability than univariate individual gene analysis (for details, see ref. ${ }^{31}$ ). As above, the Hallmark gene set collection was used. Significance was set at FDR q $<0.05$. TcGSA was employed separately for all patients, controls, responders and non-responders. Data at T3 from two patients not following the recovery sleep protocol were excluded, while 8 participants had dropped out.

\section{Results}

Gene-based analysis

In all patients [M1], 4071 (2083 up, 1988 down) genes were significantly differentially expressed after SD. Significant differential changes in gene expression after SD between responders and non-responders [M2] were observed in 360 genes [150 up, 210 down] and in patients vs. controls [M3] in 495 genes [248 up, 247 down].

Table 2 shows top differentially expressed genes for these models. Tables S1.1-S1.3 show the number of genes differentially expressed, upregulated and downregulated for all models, and detailed lists of differentially expressed genes.

At baseline, no genes were significantly differentially expressed between patients/controls and responders/nonresponders (Table S1.4).

\section{Target examination of differential expression of circadian genes}

We observed significant differential expression of circadian genes in models M1, M2 and M3. (Table 3, for more details, see Tables S2.1-3). Baseline differences in circadian genes between responders/non-responders, and patients/controls did not reach FDR $\mathrm{q}<0.05$, but achieved nominal significance (see Table S2.4).

Monte Carlo simulations confirmed that significantly more circadian genes were differentially expressed than random gene sets of the same size (see SI).

\section{Gene set enrichment analysis}

GSEA notably found enrichment in immune response related pathways (see Tables S3.1-3).

Table 2 Top 10 differentially expressed genes for each model (T2 vs. T1).

\begin{tabular}{|c|c|c|c|c|c|c|c|c|}
\hline \multicolumn{3}{|l|}{ Patients [M1] } & \multicolumn{3}{|c|}{ Responders vs. Non-responders [M2] } & \multicolumn{3}{|c|}{ Patients vs. Controls [M3] } \\
\hline Gene symbol & Estimate & P-val FDR & Gene symbol & Estimate & P-val FDR & Gene symbol & Estimate & P-val FDR \\
\hline TSPAN2 & 0.554523443 & 7.53143E-19 & DNER & 0.19414884 & 0.000170023 & ERN1 & 0.30163938 & 0.003013102 \\
\hline KLF6 & 0.162981029 & 2.03907E-16 & LPCAT2 & 0.32653286 & 0.000170023 & $\mathrm{EZH} 2$ & 0.38906547 & 0.003013102 \\
\hline MAK & 0.476984074 & $1.20412 \mathrm{E}-15$ & SLC10A5 & -0.62752802 & 0.000170023 & SLC44A1 & 0.27499289 & 0.003013102 \\
\hline ANTXR2 & 0.199530713 & 4.42924E-15 & PCID2 & -0.300504 & 0.000209042 & MEMO1 & 0.43613957 & 0.003013102 \\
\hline TMEM43 & 0.19700767 & 5.80393E-15 & TESC-AS1 & -0.3069843 & 0.001180095 & UTP11L & 0.4553397 & 0.003013102 \\
\hline TREM1 & 0.300131404 & $1.1734 \mathrm{E}-14$ & BECN1 & 0.21458155 & 0.001180095 & NSFL1C & 0.2298895 & 0.003013102 \\
\hline LRRFIP1 & 0.178631499 & $1.1734 \mathrm{E}-14$ & IFT74 & -0.43960958 & 0.001375265 & PCTP & 0.30731503 & 0.003013102 \\
\hline NHSL2 & 0.253418357 & 2.50255E-14 & ZNF790 & -0.29221374 & 0.002668032 & ZBTB16 & 0.53874494 & 0.003013102 \\
\hline ARHGEF40 & 0.32560393 & 3.23301E-14 & GSR & 0.17871771 & 0.00297074 & $\mathrm{CISH}$ & -0.31446529 & 0.003456021 \\
\hline SIPA1L1 & 0.228865158 & $3.2956 \mathrm{E}-14$ & LINC01125 & -0.16389193 & 0.00297074 & TRAV4 & -0.4598706 & 0.003456021 \\
\hline
\end{tabular}


Table 3 Differential expression in circadian genes after SD.

\begin{tabular}{|c|c|c|c|c|}
\hline & & Patients [M1] & Responders vs. Non-responders [M2] & Patients vs. Controls [M3] \\
\hline & Gene & T2 vs. T1 & T2 vs. T1 & T2 vs. T1 \\
\hline \multirow{11}{*}{$\begin{array}{c}\text { Traditional Clock } \\
\text { Genes }\end{array}$} & & $P-\operatorname{val}(\beta)$ & $P-\operatorname{val}(\beta)$ & $P-\operatorname{val}(\beta)$ \\
\hline & PER1* & NS & 0.083 FDR $(0.27)$ & 0.045 FDR $(0.32)$ \\
\hline & NR1D2* & NS & NS & NS \\
\hline & PER3 & 0.0063 FDR $(-0.11)$ & NS & 0.065 FDR $(-0.22)$ \\
\hline & NR1D1 & 0.0014 FDR $(-0.12)$ & 0.035 unc $(-0.14)$ & 0.036 FDR $(-0.27)$ \\
\hline & PER2 & 0.038 unc $(-0.059)$ & NS & NS \\
\hline & ARNTL & 0.017 FDR (0.059) & 0.033 unc (0.096) & NS \\
\hline & NPAS2 & NS & NS & 0.0054 unc $(-0.18)$ \\
\hline & CRY2 & NS & NS & NS \\
\hline & CRY1 & NS & NS & NS \\
\hline & CLOCK & NS & 0.029 unc $(-0.15)$ & NS \\
\hline \multirow{13}{*}{$\begin{array}{c}\text { Circadian } \\
\text { Genes }\end{array}$} & DDIT4 & $1.24 \mathrm{e}-04$ FDR $(-0.32)$ & NS & NS \\
\hline & CLEC4E & 0.0023 FDR $(0.20)$ & 0.0483 FDR $(0.42)$ & 0.018 FDR (0.48) \\
\hline & FKBP5 & 0.037 unc $(0.088)$ & NS & 0.028 FDR $(0.38)$ \\
\hline & DAAM2 & 0.037 FDR $(0.069)$ & 0.020 unc $(0.14)$ & NS \\
\hline & TPST1 & 1.85e-04 FDR (0.23) & NS & 0.025 FDR $(0.44)$ \\
\hline & IL13RA1 & NS & 0.0047 unc (0.19) & NS \\
\hline & SMAP2 & 1.16e-5 FDR (0.12) & 0.0390 FDR $(0.18)$ & 0.030 FDR $(0.18)$ \\
\hline & HNRNPDL & $1.32 \mathrm{e}-04$ FDR $(-0.10)$ & 0.0173 unc $(-0.13)$ & 0.035 FDR $(-0.18)$ \\
\hline & FOSL2 & 2.23e-06 FDR (0.19) & 0.0046 unc $(0.22)$ & 0.0046 unc (0.22) \\
\hline & FLT3 & NS & NS & NS \\
\hline & CDC42EP2 & 4.19e-04 FDR (0.18) & 0.0071 unc $(0.26)$ & NS \\
\hline & TMEM88 & 9.50e-05 FDR (0.21) & NS & 0.023 unc $(0.23)$ \\
\hline & RBM3 & 7.12e-11 FDR $(-0.21)$ & NS & 0.024 unc $(-0.14)$ \\
\hline
\end{tabular}

Bold text indicates FDR $q<0.05$

Blue $=$ downregulation, Red $=$ upregulation

FDR false discovery rate, NS not significant, unc uncorrected

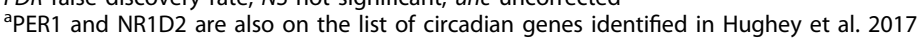

For M1, M2 and M3, 12, 23 and 11 gene sets were significantly positively enriched, respectively (FDR $\mathrm{q}<$ $0.05)$. The $T N F \alpha$ signalling via $N F K \beta$ gene set had the strongest positive enrichment in all models, while Inflammatory Response was also consistently among the significantly positively enriched gene sets.

Given the enrichment observed in Tumor Necrosis Factor Alpha (TNF $\alpha)$ and immune pathways, selected genes prominent in immune processes ${ }^{26}$ were further examined (Table S4).

\section{Time-course gene set analysis}

TcGSA results mirrored and extended gene-based analysis results:

In responders, 48 gene sets varied significantly (the model for one gene set, G2M Checkpoint, did not converge) (see Fig. 2a). Descriptively, in comparison to T1, responders showed a spectrum of differential gene expression at $\mathrm{T} 2$, with strong upregulation observed (TNF $\alpha$ Signalling via NFK $\beta$, IL6-JAK-STAT3-Signaling, Inflammatory Response, Angiogenesis) and maintained until T3. The Interferon Gamma Response and Interferon Alpha Response gene sets showed the strongest upregulation at T3.

In non-responders, 44 gene sets varied significantly (the model for one gene set, Allograft Rejection, did not converge) (see Fig. 2b), the majority of gene sets were downregulated at T2, with upregulation of the abovementioned gene sets only observed at $\mathrm{T} 3$.
In patients, expression was observed to vary significantly in 49 gene sets (see Fig. 2c). Descriptively, upregulation was observed in immune system related gene sets in two main patterns; (1) strong upregulation at T2, sustained but weakening at T3 (i.e. Inflammatory Response, IL6-JakStat3-Signalling and TNF $\alpha$ Signalling via NFK $\beta$ ) and (2) light upregulation at T2 followed by stronger upregulation at T3 (e.g., Interferon Gamma Response, Interferon Alpha Response).

In controls, 21 gene sets varied significantly (see Fig. 2d). Descriptively, at T3, immune/inflammation related gene sets were upregulated (e.g. Inflammatory Response, IL6Jak-Stat3-Signalling, TNF $\alpha$ Signalling via NFK $\beta$ ). The majority of other gene sets were downregulated at T2, followed by upregulation at T3.

Tables S5.1-4 list significant TcGSA gene sets.

\section{Discussion}

Here, we report the first longitudinal transcriptomewide study of SD treatment in major mood disorder patients suffering from a major depressive episode. Widespread differential gene expression was observed after SD; circadian genes were differentially expressed, and enrichment in pathways related to immune function, inflammatory response and sleep regulation was observed.

It has been hypothesized that SD exerts its antidepressant effect by resetting disturbed clock gene functioning thought to be a feature of depression ${ }^{10}$. Consequently, we examined changes in expression of 


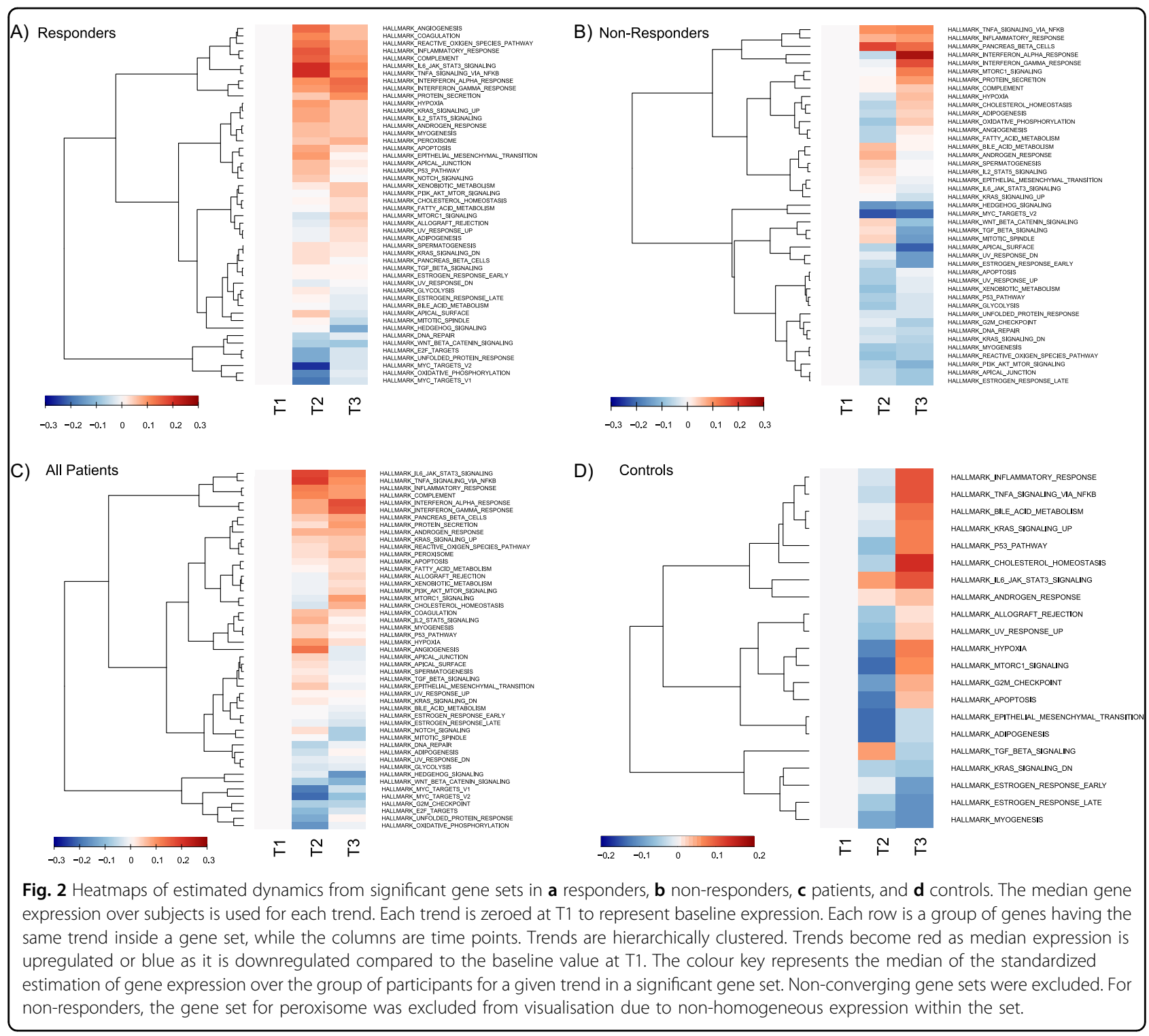

circadian genes. All individuals, i.e. patients and controls, showed significant differential expression of circadian genes after SD. In responders, many significant gene expression changes were observed while in nonresponders only a few nominally significant changes were found. The following observation may be of interest: three circadian genes, PER1, CLEC4E and SMAP2 (FDR q $=0.083$ approaching significance, 0.048, and 0.039, respectively) showed strong differential gene expression, i.e. increased expression, in responders versus nonresponders after SD. While the roles of CLEC4E and $S M A P 2$ are yet unexplored in this context (however, see below for additional discussion of CLEC4E), similar PER1related findings have been previously reported; consistent with the present findings, PER1 expression is observed to be increased in SD responders and decreased in non- responders ${ }^{10}$. Animal studies have shown that sleep deprivation or prolonged wakefulness enhanced Per1 expression in several brain regions ${ }^{21,37}$, and that quetiapine increased Per 1 expression in the amygdala ${ }^{38}$.

The present results are interesting in light of a study in human post-mortem brain tissue of $\sim 12,000$ ranked genes according to robustness of circadian rhythmicity across six brain regions; the top ranked circadian genes in that study were ARNTL, PER2, PER3 and NR1D1 and dysregulation in MDD vs controls were observed in these genes $^{22}$. The present study observed that these same genes were the most affected (of the traditional clock genes) as a result of SD, supporting the idea that SD may act on the dysregulation of these genes in depression.

The present findings cannot yet demonstrate that clock gene dysregulation/normalization is at the core of the SD 
mechanism. However, they suggest that (genes of) the molecular clocks of responders/non-responders, as well as patients/controls respond differently to chronobiologic stimuli, which may be associated with treatment outcomes.

Among the top 10 genes most significantly differentially expressed after SD in patients [M1] were: (i) KLF6, observed to be upregulated in individuals after experimental restriction in a genome-wide association study of sleep duration ${ }^{39}$; (ii) SIPA1L1, expression of which was found to be increased in a study examining changes in military personnel at baseline and after improvement of sleep $^{40}$; (iii) $N H S L 2$, the function of which is unknown, but which is located in a genomic region found to be differentially hydroxymethylated in a study of sleep deprivation in mice ${ }^{41}$; (iv) TREM1, which has immune function ${ }^{12}$ and (v) TSPAN2, of which a study looking for gene expression changes under fluoxetine in rats found hippocampal upregulation ${ }^{42}$. Among the top 10 genes observed in responders vs non-responders [M2] is BECN1 (Beclin1)-which (together with FKBP5, see below) is shown to be involved in priming autophagic pathways to set the stage for antidepressant action ${ }^{43,44}$. Circadian rhythms of autophagic proteins, including beclin1 have been linked to sleep disturbances ${ }^{45}$. Also in the top 10 in M2 were DNER, and GSR which have functions related to immune response ${ }^{46,47}$. Between patients and controls [M3], top genes included $E Z H 2$, which is reported to have a close relationship to IL- $6^{48}$. ZBTB16 is implicated in human sleep duration ${ }^{39}$ and sleep deprivation in animal studies $^{49}$, and $\mathrm{CISH}$ is shown to be involved in immunerelated processes ${ }^{50,51}$. Among other differentially expressed genes are ones evidently associated with antidepressive intervention, such as $F K B P 5^{52}$ where we observed significant upregulation in patients vs. controls after SD (see Tables S1.1, S2.1). FKBP5 plays an important regulatory role in stress response ${ }^{52-55}$; circadian rhythm abnormalities have been linked to the stress response system, and circadian clock genes can both regulate and be regulated by rhythms of glucocorticoid release ${ }^{56}$.

Pathway analyses showed a global effect of SD on gene expression; immunological, inflammatory response and sleep regulation involved pathways were most strongly affected. These findings are of interest in light of previous gene expression studies linking immune function to $\mathrm{MDD}^{57,58}$; SD may affect pathways involved in the MDD etiology. In patients and especially responders, in the Inflammatory Response (genes related to cytokines, growth factors, cell differentiation markers and transcription factors) ${ }^{36}$, IL6-JAK-STAT3-Signalling (aberrantly hyperactivated in patients with cancer and chronic inflammatory conditions) $)^{59}$, and TNF $\alpha$ Signalling via NFK $\beta$ (cell proliferation, differentiation, apoptosis, neuroinflammation mediated cell death) gene sets, strong upregulation was observed at T2. Prior findings have shown associations between the immune system and depression, suggesting that causal pathways exist from immune dysregulation and inflammation to $\mathrm{MDD}^{24,25,60-}$ ${ }^{63}$. This raises the question of how SD and the immune system interact and whether SD counteracts and/or enhances depression-related immunological processes.

Interestingly, and in contrast to responders, mainly weaker downregulation was observed at $\mathrm{T} 2$ in nonresponders, with upregulation of immune-related gene sets only observed at T3. This differential function of immune-related processes (i.e. blunted system responsiveness) might be associated with non-response to treatment. This preliminary finding must be further explored in larger sample sizes.

Of note, downregulation of MYC targets pathways after SD was consistently observed in both responders and non-responders but not controls. $M Y C$, well known as an oncogene ${ }^{64}$, acts as a mediator and coordinator of cell behaviour which also inversely modulates the impact of the cell cycle and circadian clock on gene expression ${ }^{65}$. It has been shown that dysregulation of $M Y C$ disrupts the molecular clock by inducing dampened expression and oscillation of CLOCK-BMAL1 master circadian transcription factor ${ }^{66}$. Removal of clock repression by $M Y C$ may play a role in the interaction of SD with depression and further investigation of these potential mechanisms is warranted.

Sleep and immunity are connected by anatomical and physiological bases ${ }^{67}$. The role of cytokines in the brain is complex and remains to be fully understood; while a full discussion of their roles is beyond the scope of this work, Table S4 shows cytokines and substances which have been implicated in both immune and sleep regulatory processes $^{26}$. The present findings, observing upregulation after SD, support reports linking levels of proinflammatory cytokines to depression and suggesting involvement in disease pathogenesis ${ }^{26,68,69}$.

One important clue to SD response may lie in the observed TNF $\alpha$ expression patterns. TNF $\alpha$ is a proinflammatory cytokine controlling expression of inflammatory genetic networks; in addition to many immunerelated functions in the brain, it influences whole organism function, including sleep regulation ${ }^{26,61}$. After SD, patients had significantly decreased expression of TNF compared to controls (FDR $\mathrm{q}=0.01$ ); upregulation was observed in controls and downregulation in patients. It should be noted here that the sample sizes were unbalanced, potentially introducing bias in the result; however, upregulated TNF in controls is consistent with reports of sleep deprivation-induced increases in TNFa levels in healthy people $\mathrm{e}^{70}$. The decrease observed in patients, and especially responders, may inform the mechanism of SD response-increased TNF $\alpha$ concentrations are reported to 
be a marker of depression and TNF $\alpha$ administration is reported to induce depressive symptoms ${ }^{71}$.

Sleep-wake cycles are accompanied by changes in circulating immune cell numbers and disturbing the circadian cycle affects immune response ${ }^{72,73}$. Acute SD reportedly affects diurnal rhythmicity of cells, mirroring the immediate immune stress response ${ }^{74,75}$. Consistent with a stress-like response, upregulation of expression of $I L 6$ and $I L 1 B$, thought to be involved in depression ${ }^{76}$ and regulation of circadian rhythm/sleep homeostasis ${ }^{13,69,77}$, was observed after SD. Given the tight coupling between circadian and immune systems, it may be that like the 'resetting' of circadian genes, the altered rhythmicity of the stress immune response system in depression is transiently normalized, leading to antidepressant effects; chronotherapeutic approaches that extend SD effects may be prolonging this normalization ${ }^{21}$.

In support of the idea of involvement of stress response associated with SD is the fact that several of the differentially expressed genes are known to be driven by glucocorticoid signalling. One possibility is that differential expression observed has been induced by stress responses associated with SD; it is not possible in the present protocol to disentangle stress and SD. For example, while as indicated above CLEC4E is not explored in the SD context, it is known to be involved in immune function and also to be driven by glucocorticoids and is thus related to the stress response ${ }^{78}$. Animal studies have shown that by removing glucocorticoid signalling via adrenalectomy, PER1, for example, was no-longer affected by SD, and responds to the stress induced by $\mathrm{SD}$ rather than $\mathrm{SD}$ itself $^{79}$.

There are several limitations to our study. The sample sizes of non-responders and controls were limited, with less power potentially contributing to the lower number of significant genes. The differences in sample sizes might furthermore create bias in the analyses of differential gene expression. While the statistical approach (linear mixed models) used in the single-gene analysis and underlying TcGSA is robust against unbalancedness and missing data, caution should still be exercised when considering the comparative results, as mentioned above. Next, although blood was sampled once per day on three consecutive days, a significant but manageable load for patients, the amount of data is sparse for statistical estimation. To better leverage longitudinal data and methods, a denser sampling scheme (e.g. every few hours) will be required to attain a more refined understanding of underlying SD mechanisms. It should be noted that the recovery sleep episode was a total of $7 \mathrm{~h}$ in length (i.e. $1800-0100 \mathrm{~h}$ ) after a $36 \mathrm{~h}$ homeostatic buildup of sleep pressure; this may not be long enough for homeostasis to recover and thus SD could have still had an effect on gene expression at T3. However, it is still unclear how circadian and homeostatic factors interact to regulate sleep processes ${ }^{80}$ and to address this in more detail, a different experimental design would have been required. Also, while food intake was not given special emphasis in this study, timing of food intake can be an entrainment signal for the circadian $\operatorname{clock}^{81}$. In the present study, some participants had small or simple meals (i.e. bread) and food intake was not restricted; possible effects cannot be excluded in the present design. Another direction, which would be informative about circadian phase, would be to include a marker of the central clock phase, e.g. melatonin secretion, also sampled at high temporal resolution. The sample studied was a naturalistic sample recruited from consecutive admissions and treated following standardized clinical guidelines. Medication regimens were tailored to the individual based on specific need, resulting in a variety of therapies used. While the effects of particular medications were not examined, to control for effects on results of the study, as an inclusion criterion, it was stipulated that the patient had to be on stable medication for at least 5 days prior to SD. Given the variety of medications used it was not possible to test for associations with drug response with sufficient statistical power. However, there was no apparent difference in substance class across response status. Nevertheless, robust effects of SD were observed, perhaps in part attributable to the consistent methodology for applying $\mathrm{SD}$ treatment in a relatively large cohort for SD. Considering the fact that depression is a heterogeneous phenotype accompanied by a heterogeneous immunophenotype $^{61}$, means that even larger sample sizes will be needed to substantiate the present findings. Finally, we investigated peripheral tissue transcriptome-wide expression changes; although an easily obtained, valuable proxy, the correlation of expression in the blood with expression in the brain, where depression is thought to act, is imperfect and requires further study ${ }^{82}$. On the other hand, it is precisely these cells that best represent the current status of the immune system and the inflammatory response, the gene expression of which appears at the centre of SD. In addition, the crosstalk of the organs and biorhythm tuning is also mediated via the blood system.

Our findings affirm and emphasize the close relationship between circadian, immune and sleep systems in depression at the transcriptomic level, but the directionality of cause-effect remains unclear. Circadian, immune and sleep dysregulation may precede, accompany or come as a result of depression; they now represent targets for treatment, which have the ability to influence clinical outcomes. Closer investigation of these systems with larger sample sizes and denser, longer-term sampling schemes will be key to disentangling and understanding the multi-level interactions occurring. 


\section{Acknowledgements}

This work has been supported by the Deutsche Forschungsgemeinschaft (DFG), SCHW 1768/1-1. A.M-L. acknowledges support from the German Federal Ministry of Education and Research (BMBF, grants 01ZX1314G, 01GS08147, 01EF1803A, 255156212 CRC 1158 subproject B09) and the Ministry of Science, Research and the Arts of the State of Baden-Wuerttemberg, Germany (MWK, grants 42-04HV.MED(16)/16/1, 42-04HV.MED(16)/27/1). M.R. was supported by the BMBF through grants within the e:Med research program 01ZX1314G. This work was also supported by BMBF/DLR grant 01EW1904. J.C.F. was supported by BMBF/PTJ grant 031L0190A. This manuscript was uploaded to the preprint server bioRxiv.

\section{Author details}

'Department of Genetic Epidemiology in Psychiatry, Central Institute of Mental Health, Medical Faculty Mannheim, University of Heidelberg, Mannheim, Germany. ${ }^{2}$ Department of Psychiatry and Psychotherapy, Central Institute of Mental Health, Medical Faculty Mannheim, University of Heidelberg, Mannheim, Germany. ${ }^{3}$ Department of Child and Adolescent Psychiatry and Psychotherapy, Central Institute of Mental Health, Medical Faculty Mannheim, University of Heidelberg, Mannheim, Germany. ${ }^{4}$ Medical Research Center, Medical Faculty Mannheim, University of Heidelberg, Mannheim, Germany. ${ }^{5}$ Department of Translational Research in Psychiatry, Max Planck Institute of Psychiatry, Munich, Germany. ${ }^{6}$ Munich Cluster of Systems Biology, SyNergy, Munich, Germany. ${ }^{7}$ Institute of Translational Medicine, University of Liverpool, Liverpool, United Kingdom. ${ }^{8}$ Department of Anaesthesiology and Operative Intensive Care, University Hospital Mannheim, Medical Faculty Mannheim, University of Heidelberg, Mannheim, Germany

\section{Conflict of interest}

A.M-L. has received consultant fees from Boehringer Ingelheim, Brainsway, Elsevier, Lundbeck Int. Neuroscience Foundation and Science Advances. All other authors report no biomedical financial interests or potential conflicts of interest.

\section{Publisher's note}

Springer Nature remains neutral with regard to jurisdictional claims in published maps and institutional affiliations.

Supplementary Information accompanies this paper at (https://doi.org/ 10.1038/541398-019-0671-7).

Received: 31 May 2019 Revised: 13 November 2019 Accepted: 22 November 2019

Published online: 18 December 2019

\section{References}

1. Gillin, J. C. The sleep therapies of depression. Prog. Neuropsychopharmacol. Biol. Psychiatry 7, 351-364 (1983).

2. Wu, J. C. \& Bunney, W. E. The biological basis of an antidepressant response to sleep deprivation and relapse: review and hypothesis. Am. J. Psychiatry 147, 14-21 (1990).

3. Benedetti, F., Barbini, B., Colombo, C. \& Smeraldi, E. Chronotherapeutics in a psychiatric ward. Sleep. Med. Rev. 11, 509-522 (2007).

4. Benedetti, F. \& Colombo, C. Sleep deprivation in mood disorders. Neuropsychobiology 64, 141-151 (2011).

5. Boland, E. M. et al. Meta-analysis of the antidepressant effects of acute sleep deprivation. J. Clin. Psychiatry 78, e1020-e1034 (2017).

6. Winkler, D. et al. Usage of therapeutic sleep deprivation: a survey in psychiatric hospitals in Austria, Germany, and Switzerland. Behav. Sleep. Med. 17, 713-20. (2019).

7. Wolf, E. et al. Synaptic plasticity model of therapeutic sleep deprivation in major depression. Sleep. Med. Rev. 30, 53-62 (2016).

8. Borbély, A. A. \& Wirz-Justice, A. Sleep sleep deprivation and depression. A hypothesis derived from a model of sleep regulation. Hum. Neurobiol. 1, 205-210 (1982).

9. Borbély, A. A., Daan, S., Wirz-Justice, A. \& Deboer, T. The two-process model of sleep regulation: a reappraisal. J. Sleep. Res. 25, 131-43. (2016).
10. Bunney, B. G. \& Bunney, W. E. Mechanisms of rapid antidepressant effects of sleep deprivation therapy: clock genes and circadian rhythms. Biol. Psychiatry 73, 1164-1171 (2013).

11. Lavebratt, C. et al. CRY2 is associated with depression. PLoS ONE 5, e9407 (2010).

12. Archer, S. N. et al. Mistimed sleep disrupts circadian regulation of the human transcriptome. Proc. Natl Acad. Sci. USA 111, E682-E691 (2014).

13. Moller-Levet, C. S. et al. Effects of insufficient sleep on circadian rhythmicity and expression amplitude of the human blood transcriptome. Proc. Natl Acad. Sci. USA 110, E1132-E1141 (2013).

14. Thimgan, M. S. et al. Cross-translational studies in human and Drosophila identify markers of sleep loss. PLoS ONE 8, e61016 (2013).

15. Lech, K. et al. Dissecting daily and circadian expression rhythms of clockcontrolled genes in human blood. J. Biol. Rhythms 31, 68-81 (2016).

16. Wisor, J. P. et al. A role for cryptochromes in sleep regulation. BMC Neurosci. 3, 20 (2002).

17. Mongrain, V., La Spada, F., Curie, T. \& Franken, P. Sleep loss reduces the DNAbinding of BMAL1, CLOCK, and NPAS2 to specific clock genes in the mouse cerebral cortex. PLoS ONE 6, e26622 (2011).

18. Laposky, A. et al. Deletion of the mammalian circadian clock gene BMAL1/ Mop3 alters baseline sleep architecture and the response to sleep deprivation. Sleep 28, 395-409. (2005).

19. Pellegrino, R. et al. Whole blood genome-wide gene expression profile in males after prolonged wakefulness and sleep recovery. Physiol. Genomics. 44, 1003-1012 (2012).

20. Watson, N. F. et al. Transcriptional signatures of sleep duration discordance in monozygotic twins. Sleep 40, 1-8 (2017). https://doi.org/10.1093/sleep/ zsw019.

21. Bunney, B. G. et al. Circadian dysregulation of clock genes: clues to rapid treatments in major depressive disorder. Mol. Psychiatry 20, 48-55 (2015).

22. $L i, J$. Z. et al. Circadian patterns of gene expression in the human brain and disruption in major depressive disorder. Proc. Natl Acad. Sci. USA 110 9950-9955 (2013).

23. Dallaspezia, S. \& Benedetti, F. Sleep deprivation therapy for depression. Curr. Top. Behav. Neurosci. 25, 483-502 (2015).

24. Jansen, R. et al. Gene expression in major depressive disorder. Mol. Psychiatry 21, 339-47. (2016).

25. Mostafavi, S. et al. Type I interferon signaling genes in recurrent major depression: increased expression detected by whole-blood RNA sequencing. Mol. Psychiatry 19, 1267-1274 (2014).

26. Rockstrom, M. D. et al. Tumor necrosis factor alpha in sleep regulation. Sleep. Med. Rev. 40, 69-78 (2018).

27. Labrecque, N. \& Cermakian, N. Circadian clocks in the immune system. J. Biol. Rhythms 30, 277-290 (2015).

28. Cermakian, $\mathrm{N}$. et al. Crosstalk between the circadian clock circuitry and the immune system. Chronobiol. Int. 30, 870-88. (2013).

29. Zielinski, M. R. \& Krueger, J. M. Sleep and innate immunity. Front. Biosci. 3, 632-642 (2011).

30. Trautmann, N. et al. Response to therapeutic sleep deprivation: a naturalistic study of clinical and genetic factors and post-treatment depressive symptom trajectory. Neuropsychopharmacology 43, 2572-2577 (2018).

31. Hejblum, B. P., Skinner, J. \& Thiebaut, R. Time-course gene set analysis for longitudinal gene expression data. PLoS Comput Biol. 11, e1004310 (2015).

32. Dai, J. L. Serial analyses of gene expression (SAGE). Methods Mol. Med. 103 161-174 (2005).

33. Hughey, J. J. Machine learning identifies a compact gene set for monitoring the circadian clock in human blood. Genome Med. 9, 19 (2017).

34. Subramanian, A. et al. Gene set enrichment analysis: a knowledge-based approach for interpreting genome-wide expression profiles. Proc. Natl Acad. Sci. USA 102, 15545-15550 (2005).

35. Plaisier, S. B., Taschereau, R., Wong, J. A. \& Graeber, T. G. Rank-rank hypergeometric overlap: identification of statistically significant overlap between gene-expression signatures. Nucleic Acids Res. 38, e169 (2010).

36. Liberzon, A. et al. The Molecular Signatures Database (MSigDB) hallmark gene set collection. Cell Syst. 1, 417-25. (2015).

37. Zhang, B., Gao, Y., Li, Y., Yang, J. \& Zhao, H. Sleep deprivation influences circadian gene expression in the lateral habenula. Behav. Neurol. 2016, 7919534 (2016)

38. Moriya, S. et al. Effect of quetiapine on Per1, Per2, and Bmal1 clock gene expression in the mouse amygdala and hippocampus. J. Pharm. Sci. 125, 329-332 (2014). 
39. Ollila, H. M. et al. Genome-wide association study of sleep duration in the Finnish population. J. Sleep. Res. 23, 609-18. (2014).

40. Livingston, W. S. et al. Improved sleep in military personnel is associated with changes in the expression of inflammatory genes and improvement in depression symptoms. Front. Psychiatry 6, 59 (2015).

41. Massart, R. et al. The genome-wide landscape of DNA methylation and hydroxymethylation in response to sleep deprivation impacts on synaptic plasticity genes. Transl. Psychiatry 4, e347 (2014).

42. Kroeze, Y. et al. Long-term consequences of chronic fluoxetine exposure on the expression of myelination-related genes in the rat hippocampus. Transl. Psychiatry 5, e642 (2015).

43. Gassen, N. C., Hartmann, J., Schmidt, M. V. \& Rein, T. FKBP5/FKBP51 enhances autophagy to synergize with antidepressant action. Autophagy 11, 578-580 (2015).

44. Wirawan, E. et al. Beclin1: a role in membrane dynamics and beyond. Autophagy 8, 6-17 (2012).

45. Post, R. M., Kotin, J. \& Goodwin, F. K. Effects of sleep deprivation on mood and central amine metabolism in depressed patients. Arch. Gen. Psychiatry 33, 627-632 (1976)

46. Greene, M., Lai, Y., Baella, N., Dalmau, J. \& Lancaster, E. Antibodies to Delta/ notch-like epidermal growth factor-related receptor in patients with anti-Tr, paraneoplastic cerebellar degeneration, and Hodgkin lymphoma. JAMA Neurol. 71, 1003-1008 (2014).

47. Yan, J. et al. Glutathione reductase facilitates host defense by sustaining phagocytic oxidative burst and promoting the development of neutrophil extracellular traps. J. Immunol. 188, 2316-2327 (2012).

48. Murphy, T. M. et al. Anxiety is associated with higher levels of global DNA methylation and altered expression of epigenetic and interleukin- 6 genes. Psychiatr. Genet. 25, 71-78 (2015).

49. Wang, H., Liu, Y., Briesemann, M. \& Yan, J. Computational analysis of gene regulation in animal sleep deprivation. Physiol. Genomics. 42, 427-436 (2010).

50. Ito, S. et al. Ultra-low dose interleukin-2 promotes immune-modulating function of regulatory T cells and natural killer cells in healthy volunteers. Mol. Ther. 22, 1388-95. (2014).

51. Cao, G. et al. mTOR inhibition potentiates cytotoxicity of Vgamma4 gammadelta T cells via up-regulating NKG2D and TNF-alpha. J. Leukoc. Biol. 100, 1181-1189 (2016).

52. Binder, E. B. et al. Polymorphisms in FKBP5 are associated with increased recurrence of depressive episodes and rapid response to antidepressant treatment. Nat. Genet. 36, 1319-1325 (2004).

53. Binder, E. B. The role of FKBP5, a co-chaperone of the glucocorticoid receptor in the pathogenesis and therapy of affective and anxiety disorders. Psychoneuroendocrinology 34(Suppl 1), S186-S195 (2009).

54. Lekman, M. et al. The FKBP5-gene in depression and treatment response-an association study in the Sequenced Treatment Alternatives to Relieve Depression (STAR*D) Cohort. Biol. Psychiatry 63, 1103-1110 (2008).

55. Menke, A. \& Binder, E. B. Epigenetic alterations in depression and antidepressant treatment. Dialogues Clin. Neurosci. 16, 395-404 (2014).

56. Landgraf, D., McCarthy, M. J. \& Welsh, D. K. Circadian clock and stress interactions in the molecular biology of psychiatric disorders. Cur Psychiatry Rep. 16, 483 (2014).

57. Blume, J., Douglas, S. D. \& Evans, D. L. Immune suppression and immune activation in depression. Brain Behav. Immun. 25, 221-229 (2011).

58. Leday, G. G. R. et al. Replicable and coupled changes in innate and adaptive immune gene expression in two case-control studies of blood microarrays in major depressive disorder. Biol. Psychiatry 83, 70-80 (2018).

59. Johnson, D. E., O'Keefe, R. A. \& Grandis, J. R. Targeting the IL-6/JAK STAT3 signalling axis in cancer. Nat. Rev. Clin. Oncol. 15, 234-48. (2018).

60. Liu, Y., Ho, R. C. \& Mak, A. Interleukin (IL)-6, tumour necrosis factor alpha (TNFalpha) and soluble interleukin-2 receptors (sIL-2R) are elevated in patients with major depressive disorder: a meta-analysis and meta-regression. J. Affect Disord. 139, 230-239 (2012).

61. Barnes, J., Mondelli, V. \& Pariante, C. M. Genetic contributions of inflammation to depression. Neuropsychopharmacology 42, 81-98 (2017).

62. Vogelzangs, N. et al. Association of depressive disorders, depression characteristics and antidepressant medication with inflammation. Transl. Psychiatry 2, e79 (2012).

63. Dowlati, Y. et al. A meta-analysis of cytokines in major depression. Biol. Psychiatry 67, 446-457 (2010).

64. Conacci-Sorrell, M., McFerrin, L. \& Eisenman, R. N. An overview of MYC and its interactome. Cold Spring Harb. Perspect. Med. 4, a014357 (2014).

65. Shostak, A. et al. MYC/MIZ1-dependent gene repression inversely coordinates the circadian clock with cell cycle and proliferation. Nat. Commun. 7, 11807 (2016).

66. Altman, B. J. et al. MYC disrupts the circadian clock and metabolism in cancer cells. Cell Metab. 22, 1009-1019 (2015).

67. Almeida, C. M. \& Malheiro, A. Sleep, immunity and shift workers: a review. Sleep. Sci. 9, 164-168 (2016).

68. Krueger, J. M. et al. A physiologic role for IL-1 beta and TNF-alpha. Ann. N. Y. Acad. Sci. 856, 148-159 (1998).

69. Tsai, S. J. Effects of interleukin-1beta polymorphisms on brain function and behavior in healthy and psychiatric disease conditions. CytokineGrowth Factor Rev. 37, 89-97 (2017).

70. Chennaoui, M. et al. Effect of one night of sleep loss on changes in tumor necrosis factor alpha (TNF-alpha) levels in healthy men. Cytokine 56, 318-324 (2011).

71. Pollak, Y. \& Yirmiya, R. Cytokine-induced changes in mood and behaviour: implications for 'depression due to a general medical condition', immunotherapy and antidepressive treatment. Int. J. Neuropsychopharmacol. 5 389-399 (2002).

72. Kervezee, L., Cuesta, M., Cermakian, N. \& Boivin, D. B. Simulated night shift work induces circadian misalignment of the human peripheral blood mononuclear cell transcriptome. Proc. Natl Acad. Sci. USA 115, 5540-5545 (2018).

73. Aho, V. et al. Partial sleep restriction activates immune response-related gene expression pathways: experimental and epidemiological studies in humans. PLOS ONE 8, e77184 (2013).

74. Ackermann, K. et al. Effect of sleep deprivation on rhythms of clock gene expression and melatonin in humans. Chronobiol. Int. 30, 901-909 (2013).

75. Ackermann, K. et al. Diurnal rhythms in blood cell populations and the effect of acute sleep deprivation in healthy young men. Sleep 35, 933-940 (2012).

76. Yoshimura, R. et al. Plasma levels of interleukin-6 and selective serotonin reuptake inhibitor response in patients with major depressive disorder. Hum. Psychopharmacol. 28, 466-470 (2013).

77. Cavadini, G. et al. TNF-alpha suppresses the expression of clock genes by interfering with E-box-mediated transcription. Proc. Natl Acad. Sci. USA 104, 12843-12848 (2007).

78. Laing, E. E. et al. Blood transcriptome based biomarkers for human circadian phase. elife 6, e20214 (2017).

79. Mongrain, V. et al. Separating the contribution of glucocorticoids and wakefulness to the molecular and electrophysiological correlates of sleep homeostasis. Sleep 33, 1147-1157 (2010).

80. Cirelli, C. \& Tononi, G. Linking the need to sleep with synaptic function. Science 366, 189-90. (2019).

81. Cox, K. H. \& Takahashi, J. Circadian clock genes and the transcriptional architecture of the clock mechanism. J. Mol. Endocrinol. 63, R93-R102 (2019).

82. Sullivan, P. F., Fan, C. \& Perou, C. M. Evaluating the comparability of gene expression in blood and brain. Am. J. Med. Genet. B Neuropsychiatr. Genet. 141B, 261-268 (2006). 